\title{
Mini-laparoscopic Sentinel Node Detection in Endometrial Cancer: Further Reducing Invasiveness for Patients with Early-Stage Disease
}

\author{
F. Ghezzi, MD, J. Casarin, MD, and S. Uccella, MD, PhD \\ Department of Obstetrics and Gynecology, Del Ponte Hospital, University of Insubria, Varese, Italy
}

\begin{abstract}
Background. Lymphatic mapping and sentinel lymph node (SLN) biopsy have been proposed as a safer and less morbid approach than full lymphadenectomy for patients with early endometrial cancer (EC), through either cervical or corporeal dye injection. ${ }^{1-4}$ The advantage of mini-laparoscopy is a further reduction in the overall surgical trauma for the patient. This video aims to show the feasibility of SLN biopsy using a 3-mm mini-laparoscopic approach.

Methods. A 56-year-old woman with grade 2 endometrioid EC underwent mini-laparoscopic pelvic SLN detection plus extrafascial total hysterectomy and bilateral salpingooophorectomy (TLH-BSO). A two-sided superficial and deep cervical injection of indocyanine green $(2 \mathrm{~mL}$ diluted to $1.25 \mathrm{mg} / \mathrm{mL}$ ) was used for inoculation before the procedure. A 5.8- $\mathrm{mm} 0^{\circ}$ optical camera with a near-infrared highintensity light source for detection of fluorescence imaging was inserted through the umbilicus. Two ancillary 3-mm trocars were inserted suprapubically. The procedure was accomplished using only 3-mm instruments.

Results. Neither intraoperative complications nor conversion to conventional laparoscopy or open surgery occurred. The operative time was $60 \mathrm{~min}$, and the estimated blood loss was $50 \mathrm{~mL}$. SLN was detected bilaterally, and removal of the two identified nodes was achieved through meticulous dissection and preservation of the surrounding structures followed by TLH-BSO. No postoperative
\end{abstract}

Electronic supplementary material The online version of this article (doi:10.1245/s10434-015-4875-7) contains supplementary material, which is available to authorized users.

(C) Society of Surgical Oncology 2015

First Received: 8 May 2015;

Published Online: 7 October 2015

S. Uccella, MD, PhD

e-mail: stefucc@libero.it complications were registered, and the patient was discharged $24 \mathrm{~h}$ after surgery. An SLN ultrastaging exam was negative, and the final pathology showed a International Federation of Gynaecology and Obstetrics (FIGO) stage 1A G2 EC with a 2/21-mm myometrial invasion.

Conclusion. Mini-laparoscopic SLN detection plus TLHBSO is a feasible procedure that guarantees minimal surgical trauma to selected patients with early EC.

CONFLICT OF INTEREST There are no conflicts of interest.

\section{REFERENCES}

1. Kim CH, Khoury-Collado F, Barber EL, Soslow RA, Makker V, Leitao MM Jr. et al. Sentinel lymph node mapping with pathologic ultrastaging: a valuable tool for assessing nodal metastasis in lowgrade endometrial cancer with superficial myoinvasion. Gynecol Oncol. 2013;131:714-9.

2. Daraï E, Dubernard G, Bats AS, Heitz D, Mathevet P, Marret H et al. Sentinel node biopsy for the management of early-stage endometrial cancer: long-term results of the SENTI-ENDO study. Gynecol Oncol. 2015;136:54-9.

3. Ditto A, Martinelli F, Bogani G, Papadia A, Lorusso D, Raspagliesi F et al. Sentinel node mapping using hysteroscopic injection of indocyanine green and laparoscopic near-infrared fluorescence imaging in endometrial cancer staging. J Minim Invasive Gynecol. 2015;22:132-3

4. Cormier B, Rozenholc AT, Gotlieb W, Plante M, Giede C; Communities of Practice (CoP) Group of the Society of Gynecologic Oncology of Canada (GOC). Sentinel lymph node procedure in endometrial cancer: a systematic review and proposal for standardization of future research. Gynecol Oncol. 2015;138:47885. Epub 2015 Jun 3 